\title{
A convolution estimate for two-dimensional hypersurfaces
}

\section{Ioan Bejenaru, Sebastian Herr and Daniel Tataru}

\begin{abstract}
Given three transversal and sufficiently regular hypersurfaces in $\mathbb{R}^{3}$ it follows from work of Bennett-Carbery-Wright that the convolution of two $L^{2}$ functions supported of the first and second hypersurface, respectively, can be restricted to an $L^{2}$ function on the third hypersurface, which can be considered as a nonlinear version of the Loomis-Whitney inequality. We generalize this result to a class of $C^{1, \beta}$ hypersurfaces in $\mathbb{R}^{3}$, under scaleable assumptions. The resulting uniform $L^{2}$ estimate has applications to nonlinear dispersive equations.
\end{abstract}

\section{Setup and main result}

Given three coordinate hyperplanes $\Sigma_{1}, \Sigma_{2}, \Sigma_{3}$ in $\mathbb{R}^{3}$, namely

$$
\Sigma_{1}=y z-\text { plane, } \Sigma_{2}=x z-\text { plane, } \Sigma_{3}=x y-\text { plane }
$$

and smooth functions $f \in L^{p}\left(\Sigma_{1}\right), g \in L^{q}\left(\Sigma_{2}\right)$, consider estimates of the form

$$
\|f * g\|_{L^{r^{\prime}\left(\Sigma_{3}\right)}} \leq C\|f\|_{L^{p}\left(\Sigma_{1}\right)}\|g\|_{L^{q}\left(\Sigma_{2}\right)} .
$$

Since

$$
(f * g)(x, y, z)=\int f\left(y, z^{\prime}\right) g\left(x, z-z^{\prime}\right) d z^{\prime}
$$

by duality the above estimate is equivalent to

$$
\left|\int f(y, z) g(x,-z) h(x, y) d x d y d z\right| \leq C\|f\|_{L^{p}\left(\Sigma_{1}\right)}\|g\|_{L^{q}\left(\Sigma_{2}\right)}\|h\|_{L^{r}\left(\Sigma_{3}\right)} .
$$

2000 Mathematics Subject Classification: Primary: 42B35; Secondary: 47 B38.

Keywords: Transversality, hypersurface, convolution, $L^{2}$ estimate, induction on scales. 
By Hölder's inequality, we obtain the necessary and sufficient conditions $p=q=r=2$. In that case we obtain the bound

$$
\|f * g\|_{L^{2}\left(\Sigma_{3}\right)} \leq C\|f\|_{L^{2}\left(\Sigma_{1}\right)}\|g\|_{L^{2}\left(\Sigma_{2}\right)} .
$$

which is the classical Loomis-Whitney inequality in three space dimensions, see $[8]$.

The question which we address here is the following: Does the estimate (1.1) remain true if $\Sigma_{1}, \Sigma_{2}$ and $\Sigma_{3}$ are bounded subset of transversal, sufficiently smooth, and oriented surfaces in $\mathbb{R}^{3}$ ?

This question has been answered in the affirmative in [5, Proposition 7], along with a quantitative estimate, under the assumption of $C^{3}$ regularity and a local transversality condition on the surfaces. In considering this question we are motivated by problems which arise in the analysis of bilinear $X^{s, b, p}$ estimates in various nonlinear dispersive equations. Precisely, one can view the estimate (1.1) as a limiting case of the following bound:

$$
\|f g\|_{X_{\Sigma_{3}}^{0,-\frac{1}{2}, \infty}} \leq C\|f\|_{X_{\Sigma_{1}}^{0, \frac{1}{2}, 1}}\|g\|_{X_{\Sigma_{2}}^{0, \frac{1}{2}, 1}}
$$

Here $f, g$ are assumed to have Fourier support supported in a fixed unit ball, and the norms $X_{\Sigma}^{0, \frac{1}{2}, 1}$, respectively $X_{\Sigma}^{0,-\frac{1}{2}, \infty}$ are defined by

$$
\|f\|_{X_{\Sigma}^{0, \frac{1}{2}, 1}}=\sum_{k=-\infty}^{0} 2^{\frac{k}{2}}\|\hat{f}(\xi)\|_{L^{2}\left(\left\{2^{k} \leq \operatorname{dist}(\xi, \Sigma) \leq 2^{k+1}\right\}\right)},
$$

respectively

$$
\|h\|_{X_{\Sigma}^{0,-\frac{1}{2}, \infty}}=\sup _{k \leq 0} 2^{-\frac{k}{2}}\|\hat{f}(\xi)\|_{L^{2}\left(\left\{2^{k} \leq \operatorname{dist}(\xi, \Sigma) \leq 2^{k+1}\right\}\right)} .
$$

By rescaling, this implies estimates on dyadic frequency scales, in the low modulation region. Bounds of this type have already appeared - at least implicitely - in the study of bilinear interactions in many semilinear equations with nontrivial resonance sets, i.e. when bilinear interactions of solutions to the linear homogeneous equation have an output near the characteristic set, which in our context means that $\Sigma_{1}+\Sigma_{2}$ has a nontrivial intersection with $\Sigma_{3}$.

For instance, in the context of Schrödinger equations we can mention [6], [2, Lemma 4.1] and [1]; there the three surfaces are (pieces of) parabolas. Other examples are the bounds for the KP-I equation considered in [7]. A large class of bilinear and multilinear estimates have been systematically studied in [9]; however, this does not include the present setup. 
In most applications to dispersive equations bilinear estimates are proved in an ad-hoc manner, taking advantage of the exact form of the surfaces $\Sigma_{1}$, $\Sigma_{2}$ and $\Sigma_{3}$. In all cases mentioned above the three surfaces have nonvanishing curvature, and one may ask which (if any) is the role played by the curvature in general. It is well known that the nonvanishing curvature plays a fundamental role in the study of nonlinear dispersive equations, as it insures good decay properties for the fundamental solution of the corresponding linear equation, as well as Strichartz and other estimates for solutions to the linear equation. On the other hand, the bound (1.1) is still valid when $\Sigma_{1}, \Sigma_{2}$ and $\Sigma_{3}$ are transversal planes. Note that the role of curvature and transversality has been clarified in [5] in a much broader context: Curvature is dispensable for the validity of estimate (1.1). However, it still is desireable to gain a better understanding of the interplay of the necessary size, regularity, transversality and curvature assumptions on the surfaces under which sharp quantitative and scaleable estimates of the type (1.1) hold true. Our main motivation are bilinear estimates with applications to the $2 \mathrm{~d}$ Zakharov system, see [3], in which case we need to analyze the interaction between two paraboloids and a cone.

Our precise set-up is the following.

Assumption 1.1. For $i=1,2,3$ there exists $0<\beta \leq 1, b>0$, and $\theta>0$ as well as ${ }^{1} \Sigma_{i}^{*} \subset \mathbb{R}^{3}$, such that $\Sigma_{i}$ is an open and bounded subset of $\Sigma_{i}^{*}$ and

(i) the oriented surface $\Sigma_{i}^{*}$ is given as

$$
\Sigma_{i}^{*}=\left\{\sigma_{i} \in U_{i} \mid \Phi_{i}\left(\sigma_{i}\right)=0, \nabla \Phi_{i} \neq 0, \Phi_{i} \in C^{1, \beta}\left(U_{i}\right)\right\},
$$

for a convex $U_{i} \subset \mathbb{R}^{3}$ such that $\operatorname{dist}\left(\Sigma_{i}, U_{i}^{c}\right) \geq \operatorname{diam}\left(\Sigma_{i}\right)$;

(ii) the unit normal vector field $\mathfrak{n}_{i}$ on $\Sigma_{i}^{*}$ satisfies the Hölder condition

$$
\sup _{\sigma, \tilde{\sigma} \in \Sigma_{i}^{*}} \frac{\left|\mathfrak{n}_{i}(\sigma)-\mathfrak{n}_{i}(\tilde{\sigma})\right|}{|\sigma-\tilde{\sigma}|^{\beta}}+\frac{\left|\mathfrak{n}_{i}(\sigma)(\sigma-\tilde{\sigma})\right|}{|\sigma-\tilde{\sigma}|^{1+\beta}} \leq b
$$

(iii) the matrix $N\left(\sigma_{1}, \sigma_{2}, \sigma_{3}\right)=\left(\mathfrak{n}_{1}\left(\sigma_{1}\right), \mathfrak{n}_{2}\left(\sigma_{2}\right), \mathfrak{n}_{3}\left(\sigma_{3}\right)\right)$ satisfies the transversality condition

$$
\theta \leq \operatorname{det} N\left(\sigma_{1}, \sigma_{2}, \sigma_{3}\right) \leq 1
$$

for all $\left(\sigma_{1}, \sigma_{2}, \sigma_{3}\right) \in \Sigma_{1}^{*} \times \Sigma_{2}^{*} \times \Sigma_{3}^{*}$.

\footnotetext{
${ }^{1}$ We will use the larger surfaces $\Sigma_{i}^{*}$ and condition (i) only to ensure the existence of a global representation of $\Sigma_{i}$ as a graph over a cube in a suitable frame. This assumption is likely to be redundant, but we will not pursue these matters here as it is irrelevant for applications.
} 


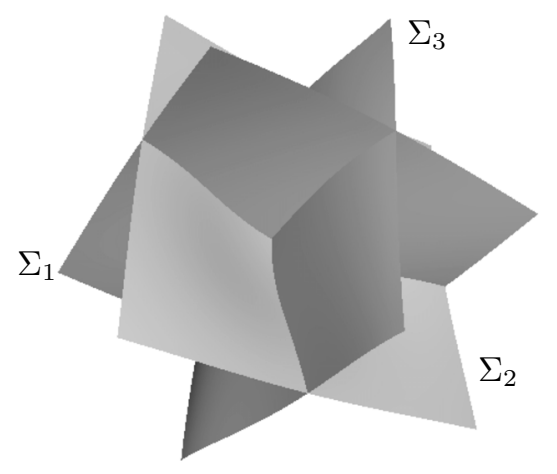

Figure 1: Three surfaces $\Sigma_{1}, \Sigma_{2}$, and $\Sigma_{3}$ in a transversal position.

We identify $f \in L^{2}\left(\Sigma_{1}\right)=L^{2}\left(\Sigma_{1}, \mu_{1}\right)-\mu_{1}$ being the surface measure on $\Sigma_{1}$ - with the distribution

$$
f(\psi)=\int_{\Sigma_{1}} f(y) \psi(y) d \mu_{1}(y), \quad \psi \in \mathcal{D}\left(\mathbb{R}^{3}\right) .
$$

For $f \in L^{2}\left(\Sigma_{1}\right), g \in L^{2}\left(\Sigma_{2}\right)$ with compact support the convolution $f * g \in$ $\mathcal{D}^{\prime}\left(\mathbb{R}^{3}\right)$ is given by

$$
(f * g)(\psi)=\int_{\Sigma_{1}} \int_{\Sigma_{2}} f(x) g(y) \psi(x+y) d \mu_{1}(x) d \mu_{2}(y), \quad \psi \in \mathcal{D}\left(\mathbb{R}^{3}\right) .
$$

A-priori this convolution is an integrable function which is only defined almost everywhere, therefore its restriction to $\Sigma_{3}$ is not well-defined. To address this issue we begin with $f \in C_{0}\left(\Sigma_{1}\right)$ and $g \in C_{0}\left(\Sigma_{2}\right)$. Then $f * g \in$ $C_{0}\left(\mathbb{R}^{3}\right)$ and has a well-defined trace on $\Sigma_{3}$. If (1.1) is proved in this case, then the trace of $f * g$ on $\Sigma_{3}$ can be defined by density for arbitrary $f \in L^{2}\left(\Sigma_{1}\right)$ and $g \in L^{2}\left(\Sigma_{2}\right)$.

Transferring the bound (1.1) from coordinate planes to the general setting of possibly curved surfaces turns out to be quite nontrivial. The reason is that the convolution has an additive structure with respect to addition in the ambient space $\mathbb{R}^{3}$, which is lost when restricting it to curved surfaces. Our first result is the following.

Theorem 1.2. Let $\Sigma_{1}, \Sigma_{2}, \Sigma_{3}$ be surfaces in $\mathbb{R}^{3}$ which satisfy Assumption 1.1 with parameters $0<\beta \leq 1, b=1$ and $\theta=\frac{1}{2}$, and diam $\Sigma_{i} \leq 1$. Then for each $f \in L^{2}\left(\Sigma_{1}\right)$ and $g \in L^{2}\left(\Sigma_{2}\right)$ the restriction of the convolution $f * g$ to $\Sigma_{3}$ is a well-defined $L^{2}\left(\Sigma_{3}\right)$-function which satisfies

$$
\|f * g\|_{L^{2}\left(\Sigma_{3}\right)} \leq C\|f\|_{L^{2}\left(\Sigma_{1}\right)}\|g\|_{L^{2}\left(\Sigma_{2}\right)},
$$

where the constant $C$ depends only on $\beta$. 
As mentioned above, in the case where the surfaces are of class $C^{3}$ and a local transversality condition is satisfied a variant of this theorem has been obtained earlier with a different proof. More precisely, estimate (1.5) can be derived from [5, Theorem 1] as in the proof of [5, Proposition 7 or Theorem 8].

For multilinear estimates with applications to nonlinear dispersive equations it is necessary to make explicit how $C$ depends on the diameter of the surfaces and on $\theta, b$ and $\beta$. The subsequent Corollaries 1.4, 1.5 and in particular 1.6 are quantitative refinements of the above Theorem which -to the best of our knowledge- are not available in the literature.

The proof of Theorem 1.2 presented here, which merely uses $C^{1, \beta}$ regularity, is based on the induction on scales argument á la Bourgain, Wolff, Tao seems to be more robust compared to the proof given in [5] in the sense that it does not require the normals to be Lipschitz. On the other hand, the induction on scales machinery has been implemented in [4] in a more general context, but the results of [4] imply our results only up to a small loss in the induction on scales procedure, see also [4, Remark 6.3]. Note that the homogeneous regularity assumption (1.3) allows us to take advantage of the isotropic scaling, which is essential for the derivation of the subsequent Corollaries.

The result (1.5) can be viewed as a weaker form of the three dimensional multilinear restriction conjecture, see [4]. Denoting

$$
\mathcal{E}_{j} f_{j}=\int_{\Sigma_{j}} e^{i x \xi} f_{j}(\xi) d \xi, \quad f_{j} \in L^{1}\left(\Sigma_{j}\right)
$$

we have

Conjecture 1.3. ([4]) Assume that $\Sigma_{1}, \Sigma_{2}$ and $\Sigma_{3}$ satisfy the transversality condition (iii) above. Then

$$
\left\|\mathcal{E}_{1} f_{1} \mathcal{E}_{2} f_{2} \mathcal{E}_{3} f_{3}\right\|_{L^{1}} \leq C\left\|f_{1}\right\|_{L^{2}\left(\Sigma_{1}\right)}\left\|f_{2}\right\|_{L^{2}\left(\Sigma_{2}\right)}\left\|f_{3}\right\|_{L^{2}\left(\Sigma_{3}\right)}
$$

With an $\epsilon$ loss this is proved in [4],

$$
\left\|\mathcal{E}_{1} f_{1} \mathcal{E}_{2} f_{2} \mathcal{E}_{3} f_{3}\right\|_{L^{1}(B(0, R))} \leq C R^{\epsilon}\left\|f_{1}\right\|_{L^{2}\left(\Sigma_{1}\right)}\left\|f_{2}\right\|_{L^{2}\left(\Sigma_{2}\right)}\left\|f_{3}\right\|_{L^{2}\left(\Sigma_{3}\right)} .
$$

Another generalization of the Loomis-Whitney inequality is given in [5]. In the context of the above restriction conjecture, the results in [5] imply that

$$
\left\|\mathcal{E}_{1} f_{1} \mathcal{E}_{2} f_{2} \mathcal{E}_{3} f_{3}\right\|_{L^{2}} \leq C\left\|f_{1}\right\|_{L^{4 / 3}\left(\Sigma_{1}\right)}\left\|f_{2}\right\|_{L^{4 / 3}\left(\Sigma_{2}\right)}\left\|f_{3}\right\|_{L^{4 / 3}\left(\Sigma_{3}\right)}
$$

which would follow from (1.6) by multilinear interpolation with the trivial $L^{\infty}$ bound for the product. 
We conclude the section with a discussion of further versions of our main result. Partitioning the three surfaces into smaller pieces and using linear changes of coordinates it is easy to allow arbitrary values for the parameters in the hypothesis of the theorem:

Corollary 1.4. Let $\Sigma_{1}, \Sigma_{2}, \Sigma_{3}$ be surfaces in $\mathbb{R}^{3}$ which satisfy Assumption 1.1 with parameters $0<\beta \leq 1, b>0, \theta>0$, and diam $\Sigma_{i} \leq R$. Then for each $f \in L^{2}\left(\Sigma_{1}\right)$ and $g \in L^{2}\left(\Sigma_{2}\right)$ the restriction of the convolution $f * g$ to $\Sigma_{3}$ is a well-defined $L^{2}\left(\Sigma_{3}\right)$-function which satisfies

$$
\|f * g\|_{L^{2}\left(\Sigma_{3}\right)} \leq C\left(R^{\beta} b, \theta\right)\|f\|_{L^{2}\left(\Sigma_{1}\right)}\|g\|_{L^{2}\left(\Sigma_{2}\right)} .
$$

Here the expression $R^{\beta} b$ appears due to isotropic scaling. While this is easy to prove, it is not so useful due to the unspecified dependence of the constant $C$ on $R^{\beta} b$ and $\theta$. A better result is contained in the next Corollary, which considers the case of three surfaces which can be placed into the context of Theorem 1.2 via a linear transformation.

Corollary 1.5. Assume that $\Sigma_{1}, \Sigma_{2}, \Sigma_{3}$ satisfy the conditions of Theorem 1.2. Let $T: \mathbb{R}^{3} \rightarrow \mathbb{R}^{3}$ be an invertible, linear map and $\Sigma_{i}^{\prime}=T \Sigma_{i}$. Then for functions $f^{\prime} \in L^{2}\left(\Sigma_{1}^{\prime}\right)$ and $g^{\prime} \in L^{2}\left(\Sigma_{2}^{\prime}\right)$ the restriction of the convolution $f^{\prime} * g^{\prime}$ to $\Sigma_{3}^{\prime}$ is a well-defined $L^{2}\left(\Sigma_{3}^{\prime}\right)$-function which satisfies

$$
\left\|f^{\prime} * g^{\prime}\right\|_{L^{2}\left(\Sigma_{3}^{\prime}\right)} \leq \frac{C}{\sqrt{d}}\left\|f^{\prime}\right\|_{L^{2}\left(\Sigma_{1}^{\prime}\right)}\left\|g^{\prime}\right\|_{L^{2}\left(\Sigma_{2}^{\prime}\right)}
$$

where

$$
d=\inf _{\sigma_{1}^{\prime}, \sigma_{2}^{\prime}, \sigma_{3}^{\prime}}\left|\operatorname{det} N^{\prime}\left(\sigma_{1}^{\prime}, \sigma_{2}^{\prime}, \sigma_{3}^{\prime}\right)\right|,
$$

$N^{\prime}\left(\sigma_{1}^{\prime}, \sigma_{2}^{\prime}, \sigma_{3}^{\prime}\right)$ is the matrix of the unit normals to $\Sigma_{i}^{\prime}$ at $\left(\sigma_{1}^{\prime}, \sigma_{2}^{\prime}, \sigma_{3}^{\prime}\right)$ and $C$ depends only on $\beta$.

We remark that the linear transformation $T$ does not explicitely appear in the estimate (1.9). Instead, the size $\frac{1}{\sqrt{d}}$ of the constant is determined only by the transversality properties of the surfaces $\Sigma_{1}^{\prime}, \Sigma_{2}^{\prime}, \Sigma_{3}^{\prime}$. Hence the best way to interpret the result in the Corollary is to say that the bound (1.9) for the surfaces $\Sigma_{1}^{\prime}, \Sigma_{2}^{\prime}, \Sigma_{3}^{\prime}$ holds whenever these surfaces are bounded, $C^{1, \beta}$ regular and uniformly transversal with respect to some linear frame.

Finally, let us state an explicit condition which guarantees that the assumptions of Corollary 1.5 are satisfied: 
Corollary 1.6. Let $\Sigma_{1}, \Sigma_{2}, \Sigma_{3}$ be surfaces in $\mathbb{R}^{3}$ which satisfy Assumption 1.1 with parameters $0<\beta \leq 1, b>0, \theta>0$, and diam $\Sigma_{i} \leq R$, so that

$$
R^{\beta} b \leq \theta .
$$

Then for each $f \in L^{2}\left(\Sigma_{1}\right)$ and $g \in L^{2}\left(\Sigma_{2}\right)$ the restriction of the convolution $f * g$ to $\Sigma_{3}$ is a well-defined $L^{2}\left(\Sigma_{3}\right)$-function which satisfies

$$
\|f * g\|_{L^{2}\left(\Sigma_{3}\right)} \leq \frac{C}{\sqrt{\theta}}\|f\|_{L^{2}\left(\Sigma_{1}\right)}\|g\|_{L^{2}\left(\Sigma_{2}\right)},
$$

where $C$ depends only on $\beta$.

Finally, we remark that the factor $\theta^{-\frac{1}{2}}$ which appears in (1.11) has also been obtained in [5, Theorem 1.2], but with an unspecified dependence of $R$ on $b$ and $\theta$.

\section{Linear changes of coordinates}

The aim of this section is to use linear transformations in order to derive Corollaries 1.5, 1.6 from Theorem 1.2. Parts of these arguments will also be useful in the proof of Theorem 1.2.

Proof of Corollary 1.5. We may assume that we have a single coordinate patch for each surface, i.e. there is a global parametrization $\varphi_{i}: \mathbb{R}^{2} \supset \Omega_{i} \rightarrow \mathbb{R}^{3}$, $\varphi_{i}\left(\Omega_{i}\right)=\Sigma_{i}$. Therefore, $\Sigma_{i}^{\prime}=T \Sigma_{i}$ is parametrized by $\varphi_{i}^{\prime}=T \varphi_{i}$. For $i=1,2,3$ we define the induced normals

$$
m_{i}(u)=\partial_{1} \varphi_{i}(u) \times \partial_{2} \varphi_{i}(u), \quad m_{i}^{\prime}(u)=\partial_{1} \varphi_{i}^{\prime}(u) \times \partial_{2} \varphi_{i}^{\prime}(u) .
$$

It is easily checked that

$$
m_{i}^{\prime}=\operatorname{det}(T)\left(T^{-1}\right)^{\top} m_{i}
$$

and therefore

$$
\begin{aligned}
\left|m_{1}^{\prime}\right|\left|m_{2}^{\prime}\right|\left|m_{3}^{\prime}\right| & =\left|\operatorname{det}\left(m_{1}^{\prime}, m_{2}^{\prime}, m_{3}^{\prime}\right)\right|\left|\operatorname{det}\left(\mathfrak{n}_{1}^{\prime}, \mathfrak{n}_{2}^{\prime}, \mathfrak{n}_{3}^{\prime}\right)\right|^{-1} \\
& =\frac{\operatorname{det}(T)^{2} \operatorname{det}\left(\mathfrak{n}_{1}, \mathfrak{n}_{2}, \mathfrak{n}_{3}\right)}{\left|\operatorname{det}\left(\mathfrak{n}_{1}^{\prime}, \mathfrak{n}_{2}^{\prime}, \mathfrak{n}_{3}^{\prime}\right)\right|}\left|m_{1}\right|\left|m_{2}\right|\left|m_{3}\right| .
\end{aligned}
$$

Let $f^{\prime} \in L^{2}\left(\Sigma_{1}^{\prime}\right), g^{\prime} \in L^{2}\left(\Sigma_{2}^{\prime}\right)$ and $h^{\prime} \in L^{2}\left(\Sigma_{3}^{\prime}\right)$ be given and it is enough to consider non-negative functions. We write $f=f^{\prime}(T \cdot)$, and

$$
\tilde{f}^{\prime}(u)=f^{\prime}\left(\varphi_{1}^{\prime}(u)\right)\left|m_{1}^{\prime}(u)\right|^{\frac{1}{2}},
$$

and similarly for $g^{\prime}, h^{\prime}$. 
With this notation and by duality, our claim is equivalent to

$$
\begin{gathered}
\left.\left|\int \tilde{f}^{\prime}(u) \tilde{g}^{\prime}(v) \tilde{h}^{\prime}(w)\right| m_{1}^{\prime}(u)\right|^{\frac{1}{2}}\left|m_{2}^{\prime}(v)\right|^{\frac{1}{2}}\left|m_{3}^{\prime}(w)\right|^{\frac{1}{2}} d \nu^{\prime}(u, v, w) \mid \leq \\
\leq \frac{C}{\sqrt{d}}\left\|\tilde{f}^{\prime}\right\|_{L^{2}\left(\Omega_{1}\right)}\left\|\tilde{g}^{\prime}\right\|_{L^{2}\left(\Omega_{2}\right)}\left\|\tilde{h}^{\prime}\right\|_{L^{2}\left(\Omega_{3}\right)}
\end{gathered}
$$

with the measure

$$
d \nu^{\prime}(u, v, w)=\delta\left(\varphi_{1}^{\prime}(u)+\varphi_{2}^{\prime}(v)-\varphi_{3}^{\prime}(w)\right) d u d v d w,
$$

where $\delta$ denotes the Dirac delta distribution at the origin in $\mathbb{R}^{3}$. Let us also define the measure

$$
d \nu(u, v, w)=\delta\left(\varphi_{1}(u)+\varphi_{2}(v)-\varphi_{3}(w)\right) d u d v d w .
$$

Using $\delta(T \cdot)=|\operatorname{det}(T)|^{-1} \delta(\cdot)$, and (2.2) we have that

$$
\left|m_{1}^{\prime}\right|^{\frac{1}{2}}\left|m_{2}^{\prime}\right|^{\frac{1}{2}}\left|m_{3}^{\prime}\right|^{\frac{1}{2}} d \nu^{\prime} \sim \frac{\left|m_{1}\right|^{\frac{1}{2}}\left|m_{2}\right|^{\frac{1}{2}}\left|m_{3}\right|^{\frac{1}{2}}}{\left|\operatorname{det}\left(\mathfrak{n}_{1}^{\prime}, \mathfrak{n}_{2}^{\prime}, \mathfrak{n}_{3}^{\prime}\right)\right|^{\frac{1}{2}}} d \nu
$$

by the transversality assumption (1.4) with $\theta=\frac{1}{2}$ on the normals to $\Sigma_{i}$. Therefore (2.3) is equivalent to

$$
\begin{gathered}
\left|\int \tilde{f}^{\prime}(u) \tilde{g}^{\prime}(v) \tilde{h}^{\prime}(w) \frac{\left|m_{1}(u)\right|^{\frac{1}{2}}\left|m_{2}(v)\right|^{\frac{1}{2}}\left|m_{3}(w)\right|^{\frac{1}{2}} d \nu(u, v, w)}{\left|\operatorname{det}\left(\mathfrak{n}_{1}^{\prime}\left(\varphi_{1}^{\prime}(u)\right), \mathfrak{n}_{2}^{\prime}\left(\varphi_{2}^{\prime}(v)\right), \mathfrak{n}_{3}^{\prime}\left(\varphi_{3}^{\prime}(w)\right)\right)\right|^{\frac{1}{2}}}\right| \\
\leq \frac{C}{\sqrt{d}}\left\|\tilde{f}^{\prime}\right\|_{L^{2}\left(\Omega_{1}\right)}\left\|\tilde{g}^{\prime}\right\|_{L^{2}\left(\Omega_{2}\right)}\left\|\tilde{h}^{\prime}\right\|_{L^{2}\left(\Omega_{3}\right)} .
\end{gathered}
$$

Observe that it follows from our assumption that the corresponding estimate for $\Sigma_{1}, \Sigma_{2}, \Sigma_{3}$, namely

$$
\begin{aligned}
& \left.\left|\int \tilde{f}^{\prime}(u) \tilde{g}^{\prime}(v) \tilde{h}^{\prime}(w)\right| m_{1}(u)\right|^{\frac{1}{2}}\left|m_{2}(v)\right|^{\frac{1}{2}}\left|m_{3}(w)\right|^{\frac{1}{2}} d \nu(u, v, w) \mid \\
& \leq C\left\|\tilde{f}^{\prime}\right\|_{L^{2}\left(\Omega_{1}\right)}\left\|\tilde{g}^{\prime}\right\|_{L^{2}\left(\Omega_{2}\right)}\left\|\tilde{h}^{\prime}\right\|_{L^{2}\left(\Omega_{3}\right)}
\end{aligned}
$$

holds true. This implies (2.4).

Proof of Corollary 1.6. Partitioning each of the three surfaces into smaller sets we strengthen the relation (1.10) to

$$
R^{\beta} b \leq 2^{-10} \theta .
$$

Consider a fixed triplet $\left(\sigma_{1}^{0}, \sigma_{2}^{0}, \sigma_{3}^{0}\right) \in \Sigma_{1} \times \Sigma_{2} \times \Sigma_{3}$. For arbitrary $\left(\sigma_{1}, \sigma_{2}, \sigma_{3}\right)$ $\in \Sigma_{1} \times \Sigma_{2} \times \Sigma_{3}$ we use the Hölder condition to compute

$$
\left|\mathfrak{n}_{i}\left(\sigma_{i}\right)-\mathfrak{n}_{i}\left(\sigma_{i}^{0}\right)\right| \leq b R^{\beta} \leq 2^{-10} \theta, \quad i=1,2,3
$$


This implies that $N\left(\sigma_{1}, \sigma_{2}, \sigma_{3}\right)$ does not vary much,

$$
\left|\operatorname{det} N\left(\sigma_{1}^{0}, \sigma_{2}^{0}, \sigma_{3}^{0}\right)-\operatorname{det} N\left(\sigma_{1}, \sigma_{2}, \sigma_{3}\right)\right| \leq 2^{-8} \theta
$$

Hence, after possibly increasing $\theta$, we may assume that on $\Sigma_{1} \times \Sigma_{2} \times \Sigma_{3}$ we have the stronger bound

$$
\theta \leq\left|\operatorname{det} N\left(\sigma_{1}, \sigma_{2}, \sigma_{3}\right)\right| \leq\left(1+2^{-8}\right) \theta .
$$

From the Hölder condition we also obtain

$$
\left|\left(\sigma_{i}-\sigma_{i}^{0}\right) \mathfrak{n}_{i}^{0}\right| \leq 2^{-10} R \theta
$$

which shows that $\Sigma_{i}$ is contained in an infinite slab of thickness $2^{-10} R \theta$ with respect to the $\mathfrak{n}_{i}^{0}$ direction. By orthogonality with respect to such slabs it suffices to prove the desired bound (1.11) in the case when the other two surfaces are contained in similar slabs,

$$
\left|\left(\sigma_{i}-\sigma_{i}^{0}\right) \mathfrak{n}_{j}^{0}\right| \leq 2^{-10} R \theta, \quad i, j=1,2,3 .
$$

We will apply Corollary 1.5 with

$$
T=R \theta\left(N^{\top}\right)^{-1}, \quad N=N\left(\sigma_{1}^{0}, \sigma_{2}^{0}, \sigma_{3}^{0}\right) .
$$

We need to show that for $\tilde{\Sigma}_{i}:=T^{-1} \Sigma_{i}$ we have the conditions (1.4) with $b=1$, (1.3) with $\theta=\frac{1}{2}$ and the size condition $\operatorname{diam}\left(\tilde{\Sigma}_{i}\right) \leq 1$. Concerning the latter we observe that

$$
T^{-1}\left(\sigma_{i}-\sigma_{i}^{0}\right)=\frac{1}{R \theta}\left(\begin{array}{c}
\mathfrak{n}_{1}^{0}\left(\sigma_{i}-\sigma_{i}^{0}\right) \\
\mathfrak{n}_{2}^{0}\left(\sigma_{i}-\sigma_{i}^{0}\right) \\
\mathfrak{n}_{3}^{0}\left(\sigma_{i}-\sigma_{i}^{0}\right)
\end{array}\right)
$$

Thus by (2.8) we obtain

$$
\left|T^{-1}\left(\sigma_{i}-\sigma_{i}^{0}\right)\right| \leq \sqrt{3} 2^{-10} \leq \frac{1}{2}
$$

therefore $\operatorname{diam}\left(\tilde{\Sigma}_{i}\right) \leq 1$. For the transversality condition, we first estimate

$$
\left\|N^{-1}\right\| \leq 2|\operatorname{det} N|^{-1}\|N\|^{2} \leq 6 \theta^{-1}
$$

This gives a bound for $T$, namely

$$
\|T\| \leq 6 R .
$$

The unit normal $\tilde{\mathfrak{n}}_{i}\left(\tilde{\sigma}_{i}\right)$ to $\tilde{\Sigma}_{i}$ in $\tilde{\sigma}_{i} \in \tilde{\Sigma}_{i}$ is given by

$$
\tilde{\mathfrak{n}}_{i}\left(\tilde{\sigma}_{i}\right)=\frac{N^{-1} \mathfrak{n}_{i}\left(T \tilde{\sigma}_{i}\right)}{\left|N^{-1} \mathfrak{n}_{i}\left(T \tilde{\sigma}_{i}\right)\right|} .
$$


By construction for $\tilde{\sigma}_{i}^{0}=T^{-1} \sigma_{i}^{0}$ we have $\tilde{\mathfrak{n}}_{i}\left(\tilde{\sigma}_{i}^{0}\right)=N^{-1} \mathfrak{n}_{i}\left(\sigma_{i}^{0}\right)=e_{i}$. By $(2.6)$ and (2.9) it follows that

$$
\left|N^{-1} \mathfrak{n}_{i}\left(T \tilde{\sigma}_{i}\right)-e_{i}\right| \leq 2^{-7}
$$

which implies that

$$
\left|\tilde{\mathfrak{n}}_{i}\left(T \tilde{\sigma}_{i}\right)-e_{i}\right| \leq 2^{-5} .
$$

This in turn yields the desired transversality condition

$$
\operatorname{det} \tilde{N}\left(\tilde{\sigma}_{1}, \tilde{\sigma}_{2}, \tilde{\sigma}_{3}\right) \geq 1 / 2 .
$$

Finally, for the Hölder condition we use (2.9) and (2.10) to compute

$$
\frac{\left|\tilde{\mathfrak{n}}_{i}(\tilde{\sigma})-\tilde{\mathfrak{n}}_{i}(\tilde{\rho})\right|}{|\tilde{\sigma}-\tilde{\rho}|^{\beta}} \leq 3\left\|N^{-1}\right\|\|T\|^{\beta} \frac{\left|\mathfrak{n}_{i}(T \tilde{\sigma})-\mathfrak{n}_{i}(T \tilde{\rho})\right|}{|T \tilde{\sigma}-T \tilde{\rho}|^{\beta}} \leq 2^{7} \theta^{-1} R^{\beta} b \leq 1
$$

which proves the desired bound for the first term in the Hölder condition (1.3) with $b=1$. The second term in (1.3) is treated in the same way.

\section{Induction on scales}

Theorem 1.2 is obtained from uniform estimates for $f * g$ thickened surfaces $\Sigma_{3}(\varepsilon)$ given by

$$
\Sigma_{3}(\varepsilon):=\left\{v \in \mathbb{R}^{3} \mid \operatorname{dist}\left(v, \Sigma_{3}\right) \leq \varepsilon\right\} .
$$

Our main technical result is the following.

Proposition 3.1. For all $\Sigma_{1}, \Sigma_{2}$, and $\Sigma_{3}$ with $\operatorname{diam}\left(\Sigma_{i}\right) \leq 1$ as in Theorem 1.2 and $f \in L^{2}\left(\Sigma_{1}\right), g \in L^{2}\left(\Sigma_{2}\right)$ and $0<\varepsilon \leq 1$ the estimate

$$
\|f * g\|_{L^{2}\left(\Sigma_{3}(\varepsilon)\right)} \leq C \sqrt{\varepsilon}\|f\|_{L^{2}\left(\Sigma_{1}\right)}\|g\|_{L^{2}\left(\Sigma_{2}\right)}
$$

holds true with a constant $C$ depending only on $\beta$.

We first show how this implies the main Theorem.

Proof of Theorem 1.2. For $f \in C_{0}\left(\Sigma_{1}\right)$ and $g \in C_{0}\left(\Sigma_{2}\right)$ we have $f * g \in$ $C_{0}\left(\mathbb{R}^{3}\right)$. Then

$$
\|f * g\|_{L^{2}\left(\Sigma_{3}\right)}=\lim _{\varepsilon \rightarrow 0} \varepsilon^{-\frac{1}{2}}\|f * g\|_{L^{2}\left(\Sigma_{3}(\varepsilon)\right)},
$$

therefore (1.5) follows from (3.2). The result extends by density to $f \in L^{2}\left(\Sigma_{1}\right)$ and $g \in L^{2}\left(\Sigma_{2}\right)$. 
The rest of this section is devoted to the proof of Proposition 3.1. By repeating the argument from the proof of Corollary 1.6 -namely a finite partition of the surfaces $\Sigma_{i}$, scaling and transforming the normals at one triplet of points to $\mathfrak{e}_{1}, \mathfrak{e}_{2}, \mathfrak{e}_{3}$ - we can reduce Proposition 3.1 to the following setup:

There are unit cubes $\Omega_{i} \subset \mathbb{R}^{2}, i=1,2,3$ centered at points $a_{i}^{0} \in \mathbb{R}^{2}$, and $C^{1, \beta}$ functions $\phi_{i}$ in the doubled cubes $2 \Omega_{i}$ so that

$$
\begin{aligned}
& \Sigma_{1}=\left\{(x, y, z) \in \mathbb{R}^{3} \mid(y, z) \in \Omega_{1}, x=\phi_{1}(y, z)\right\}, \\
& \Sigma_{2}=\left\{(x, y, z) \in \mathbb{R}^{3} \mid(x, z) \in \Omega_{2}, y=\phi_{2}(x, z)\right\}, \\
& \Sigma_{3}=\left\{(x, y, z) \in \mathbb{R}^{3} \mid(x, y) \in \Omega_{3}, z=\phi_{3}(x, y)\right\},
\end{aligned}
$$

where the functions $\phi_{i}$ satisfy

$$
\nabla \phi_{1}\left(a_{1}^{0}\right)=\nabla \phi_{2}\left(a_{2}^{0}\right)=\nabla \phi_{3}\left(a_{3}^{0}\right)=0
$$

and have small Hölder constant

$$
\sup _{w, \tilde{w} \in 2 \Omega_{i}} \frac{\left|\nabla \phi_{i}(w)-\nabla \phi_{i}(\tilde{w})\right|}{|w-\tilde{w}|^{\beta}} \leq 2^{-40} .
$$

To set up the induction on scales we allow the scale of the cubes $\Omega_{i}$ to vary from 0 to 1 . Precisely, for $0<\varepsilon \leq r \leq 1$ we denote by $C(r, \varepsilon)$ the best constant $C$ in the estimate

$$
\|f * g\|_{L^{2}\left(\Sigma_{3}(\varepsilon)\right)} \leq C \sqrt{\varepsilon}\|f\|_{L^{2}\left(\Sigma_{1}\right)}\|g\|_{L^{2}\left(\Sigma_{2}\right)}
$$

considered over all surfaces $\Sigma_{1}, \Sigma_{2}, \Sigma_{3}$ as in (3.3) with $\Omega_{i}$ cubes of size $r$ and $\phi_{i}$ satisfying (3.4) and (3.5).

For $0 \leq \varepsilon \leq r \leq R \leq 1$ we also introduce the auxiliary notation $C(R, r, \varepsilon)$ as the best constant in the estimate (3.6) over all surfaces $\Sigma_{1}, \Sigma_{2}, \Sigma_{3}$ as in (3.3) with $\Omega_{i}$ cubes of size $r$ and $\phi_{i}$ satisfying (3.5) in larger cubes $4 \Omega_{i}$ and a weaker version of (3.4), namely

$$
\left|\nabla \phi_{i}\left(a_{i}^{0}\right)\right| \leq 2^{-40}(\sqrt{3} R)^{\beta}
$$

Throughout this paper (and hence in the above definitions) we agree to the following convention: the size of a cube is half of its side-length. The reason for doing so is purely technical as it spares us from carrying a factor of 2 in some estimates.

As a starting point of our induction we establish the desired bound when $r$ is sufficiently small, depending on $\varepsilon$.

Lemma 3.2. Assume that $r \leq \varepsilon^{\frac{1}{\beta+1}}$. Then $C(r, \varepsilon) \leq 1$. 
Proof. For $f \in L^{2}\left(\Sigma_{1}\right), g \in L^{2}\left(\Sigma_{2}\right)$ and an arbitrary test function $\psi$ the convolution $(f * g)(\psi)$ can be expressed in the form

$$
\int \tilde{f}(y, z) \tilde{g}(x, \zeta-z) \psi\left(x+\phi_{1}(y, z), y+\phi_{2}(x, \zeta-z), \zeta\right) d x d y d z d \zeta
$$

where

$$
\begin{aligned}
& \tilde{f}(y, z)=f\left(\phi_{1}(y, z), y, z\right) \sqrt{1+\left|\nabla \phi_{1}(y, z)\right|^{2}} \\
& \tilde{g}(x, \zeta)=g\left(x, \phi_{2}(x, \zeta), \zeta\right) \sqrt{1+\left|\nabla \phi_{2}(x, \zeta)\right|^{2}} .
\end{aligned}
$$

Assume that $\operatorname{supp} \psi \subset \Sigma_{3}(\varepsilon)$. For every $v \in \operatorname{supp} \psi$ there is $a \in \Omega_{3}$ such that $d\left(\left(a, \phi_{3}(a)\right), v\right) \leq \varepsilon$ and $d\left(a, a_{3}^{0}\right) \leq \sqrt{3} r$. Using (3.5) we have:

$$
\left|\phi_{3}(a)-\phi_{3}\left(a_{3}^{0}\right)\right| \leq \sqrt{3} r \sup _{b \in \Omega_{3}}\left|\nabla \phi_{3}(b)\right| \leq 2^{-10}(\sqrt{3} r)^{1+\beta} \leq 2^{-8} \varepsilon
$$

Then,

$$
\operatorname{supp} \psi \subset\left\{(x, y, z)|| z-\phi_{3}\left(a_{3}^{0}\right) \mid \leq \frac{\varepsilon}{4}\right\} .
$$

Let us denote $J(\varepsilon)=\left[\phi_{3}\left(a_{3}^{0}\right)-\frac{\varepsilon}{4}, \phi_{3}\left(a_{3}^{0}\right)+\frac{\varepsilon}{4}\right]$. The Cauchy-Schwarz inequality shows that

$$
\begin{aligned}
|(f * g)(\psi)| & \leq \int\|\tilde{f}(\cdot, z)\|_{L^{2}}\|\tilde{g}(\cdot, \zeta-z)\|_{L^{2}} I(z, \zeta) d z d \zeta \\
& \leq\|\tilde{f}\|_{L^{2}}\|\tilde{g}\|_{L^{2}} \int_{J(\varepsilon)} \sup _{z} I(z, \zeta) d \zeta
\end{aligned}
$$

where

$$
I(z, \zeta):=\left(\int\left|\psi\left(x+\phi_{1}(y, z), y+\phi_{2}(x, \zeta-z), \zeta\right)\right|^{2} d x d y .\right)^{\frac{1}{2}}
$$

By the change of variables

$$
\Phi_{z, \zeta}(x, y)=\left(x+\phi_{1}(y, z), y+\phi_{2}(x, \zeta-z)\right)
$$

we obtain

$$
I(z, \zeta) \leq \sqrt{2}\|\psi(\cdot, \cdot, \zeta)\|_{L^{2}\left(\mathbb{R}^{2}\right)},
$$

because $\left|\operatorname{det} D \Phi_{z, \zeta}(x, y)\right|=\left|1-\partial_{y} \phi_{1}(y, z) \partial_{x} \phi_{2}(x, \zeta-z)\right| \geq \frac{1}{2}$.

Finally, since $z$ is in an interval of size $\frac{\varepsilon}{2}$, using again the Cauchy-Schwarz inequality we obtain

$$
\int_{J(\varepsilon)} \sup _{z} I(z, \zeta) d \zeta \leq \frac{1}{\sqrt{2}} \sqrt{\varepsilon}\|\psi\|_{L^{2}}
$$


Hence it follows that

$$
\begin{aligned}
(f * g)(\psi) & \leq \sqrt{\varepsilon}\|\tilde{f}\|_{L^{2}}\|\tilde{g}\|_{L^{2}}\|\psi\|_{L^{2}} \\
& \leq \sqrt{\varepsilon}\|f\|_{L^{2}\left(\Sigma_{1}\right)}\|g\|_{L^{2}\left(\Sigma_{2}\right)}\|\psi\|_{L^{2}\left(\Sigma_{3}(\varepsilon)\right)} .
\end{aligned}
$$

The bound $C(r, \varepsilon) \leq 1$ follows by density and duality.

The previous lemma shows that $C(r, \varepsilon)$ is finite. By the argument in the proof of Corollary 1.6 it also follows that $C(R, r, \varepsilon)$ is always finite. The next lemma allows us to bound the auxiliary variable $C(R, r, \varepsilon)$ in terms of $C(r, \varepsilon)$.

Lemma 3.3. The following estimate holds true

$$
C(R, r, \varepsilon) \leq\left(1+R^{\beta}\right) C\left(r\left(1+R^{\beta}\right)^{1+\frac{1}{\beta}}, \varepsilon\left(1+R^{\beta}\right)^{1+\frac{1}{\beta}}\right)
$$

Proof. The argument proceeds along the lines of the proof of Corollary 1.6, carefully keeping track of the scales. Let $\sigma_{i}^{0} \in \Sigma_{i}$ be the images of $a_{i}^{0} \in \Omega_{i}$. We consider the linear transformation defined by the matrix

$$
T=\lambda\left(N^{\top}\right)^{-1}, \quad N=N\left(\sigma_{1}^{0}, \sigma_{2}^{0}, \sigma_{3}^{0}\right), \quad \lambda=\left(1+R^{\beta}\right)^{-\frac{1}{\beta}}
$$

We denote $\tilde{\Sigma}_{i}=T^{-1} \Sigma_{i}$. We will show that the surfaces $\tilde{\Sigma}_{i}$ satisfy the conditions in the definition of $C\left(r\left(1+R^{\beta}\right)^{1+\frac{1}{\beta}}, \varepsilon\left(1+R^{\beta}\right)^{1+\frac{1}{\beta}}\right)$.

We denote $\tilde{\sigma}_{i}^{0}=T^{-1} \sigma_{i}^{0}$ and let $\tilde{a}_{j}^{0}$ be the projections of $\tilde{\sigma}_{i}^{0}$ on the coordinate planes, and $\tilde{\Omega}_{i}$ the corresponding projections of $\tilde{\Sigma}_{i}$. Setting $i=3$ for convenience, we also consider the full correspondence $a_{3} \rightarrow \tilde{a}_{3}$ given by

$$
\begin{aligned}
a_{3}=(x, y) \in \Omega_{3} & \rightarrow \sigma_{3}=\left(x, y, \phi_{3}(x, y)\right) \in \Sigma_{3} \\
& \rightarrow \tilde{\sigma}_{3}=T^{-1} \sigma_{3}=(\tilde{x}, \tilde{y}, \tilde{\phi}(\tilde{x}, \tilde{y})) \in \tilde{\Sigma}_{3} \\
& \rightarrow \tilde{a}_{3}=(\tilde{x}, \tilde{y}) \in \tilde{\Omega}_{3}
\end{aligned}
$$

and similarly for $i=1,2$.

By construction the matrix of the unit normals to $\tilde{\Sigma}_{i}$ at $\tilde{\sigma}_{i}^{0}$ is

$$
\tilde{N}\left(\tilde{\sigma}_{1}^{0}, \tilde{\sigma}_{2}^{0}, \tilde{\sigma}_{3}^{0}\right)=I .
$$

This implies that the condition (3.4) is satisfied for the surfaces $\tilde{\Sigma}_{i}$ at the points $\tilde{a}_{i}^{0}$.

The condition (3.7) shows that

$$
\left|\mathfrak{n}_{k}\left(\sigma_{k}^{0}\right)-\mathfrak{e}_{k}\right| \leq 2^{-10}(\sqrt{3} R)^{\beta}
$$

which leads to

$$
\|N-I\| \leq 2^{-8} R^{\beta}, \quad\left\|N^{-1}-I\right\| \leq 2^{-8} R^{\beta} .
$$


Also by (3.7) and (3.5) it follows that

$$
\left|\nabla \phi_{3}\left(a_{3}\right)\right| \leq 2^{-6} R^{\beta}, \quad a \in 4 \Omega_{3}
$$

We claim that $\tilde{\Omega}_{i}$ is contained in a cube of size $r\left(1+R^{\beta}\right)^{\frac{1}{\beta}}$ centered at $\tilde{a}_{j}^{0}$. Set $i=3$ for convenience, and consider the canonical map $T_{3}$ from $\Omega_{3}$ to $\tilde{\Omega}_{3}$, defined by $T_{3} a_{3}=\tilde{a}_{3}$. Then, by (3.9) and (3.10) the chain rule shows that

$$
\left\|D T_{3}-\lambda^{-1} I_{2}\right\| \leq 2^{-4} R^{\beta}
$$

This implies that $\tilde{\Omega}_{i}$ is contained inside a cube centered at $\tilde{a}_{i}^{0}$ which has size

$$
\tilde{r}=r\left(\lambda^{-1}+\sqrt{3} 2^{-4} R^{\beta}\right) \leq r\left(1+R^{\beta}\right)^{1+\frac{1}{\beta}}
$$

Or next goal is to establish the bound (3.5) for $\tilde{\phi}_{3}$. Define the function $\Phi_{3}(x, y, z)=z-\phi_{3}(x, y)$. Then $\tilde{\sigma}_{3} \in \tilde{\Sigma}_{3}$ iff $\tilde{\Phi}_{3}\left(\tilde{\sigma}_{3}\right):=\Phi_{3}\left(T \tilde{\sigma}_{3}\right)=0$. The implicit function theorem guarantees the existence of $\tilde{\phi}_{3}$ which satisfies $\tilde{\Phi}_{3}\left(\tilde{x}, \tilde{y}, \tilde{\phi}_{3}(\tilde{x}, \tilde{y})\right)=0$. In addition, setting $\left(N^{\top}\right)^{-1}=\left(t_{1}, t_{2}, t_{3}\right)$, we have

$$
\nabla \tilde{\phi}_{3}(\tilde{y}, \tilde{z})=-\frac{1}{t_{3} \cdot n(x, y)}\left(\begin{array}{c}
t_{1} \cdot n(x, y) \\
t_{2} \cdot n(x, y)
\end{array}\right), n(x, y)=\left(\begin{array}{c}
-\partial_{1} \phi_{3}(x, y) \\
-\partial_{2} \phi_{3}(x, y) \\
1
\end{array}\right)
$$

By (3.9) and (3.10) we obtain

$$
\left|\left(N^{\top}\right)^{-1} n(x, y)-\mathfrak{e}_{3}\right| \leq 2^{-5} R^{\beta}
$$

which after some elementary computations leads to

$$
\left|\nabla \tilde{\phi}_{3}\left(\tilde{a}_{3}^{1}\right)-\nabla \tilde{\phi}_{3}\left(\tilde{a}_{3}^{2}\right)\right| \leq\left(1+2^{-4} R^{\beta}\right)\left|\nabla \phi_{3}\left(a_{3}^{1}\right)-\nabla \phi_{3}\left(a_{3}^{2}\right)\right|
$$

On the other hand (3.11) shows that

$$
\left|\tilde{a}_{3}^{1}-\tilde{a}_{3}^{2}\right| \geq \lambda^{-1}\left(1-2^{-4} R^{\beta}\right)\left|a_{3}^{1}-a_{3}^{2}\right|
$$

Given the value of $\lambda$ it follows that

$$
\begin{aligned}
\frac{\left|\nabla \tilde{\phi}_{3}\left(\tilde{a}_{3}^{1}\right)-\nabla \tilde{\phi}_{3}\left(\tilde{a}_{3}^{2}\right)\right|}{\left|\tilde{a}_{3}^{1}-\tilde{a}_{3}^{2}\right|^{\beta}} & \leq \frac{\left(1+2^{-4} R^{\beta}\right)}{\left(1+R^{\beta}\right)\left(1-2^{-4} R^{\beta}\right)^{\beta}} \frac{\left|\nabla \phi_{3}\left(a_{3}^{1}\right)-\nabla \phi_{3}\left(a_{3}^{2}\right)\right|}{\left|a_{3}^{1}-a_{3}^{2}\right|^{\beta}} \\
& \leq 2^{-10},
\end{aligned}
$$

hence (3.5) is established for the surfaces $\tilde{\Sigma}_{i}$.

Formula (2.1), combined with (3.9), shows how the surface measures on $\Sigma_{1}$ and $\Sigma_{2}$ change:

$$
\lambda^{2}\left(1-2^{-4} R^{\beta}\right) d \tilde{\mu}_{i}\left(\tilde{\sigma}_{i}^{\prime}\right) \leq d \mu_{i}\left(\sigma_{i}^{\prime}\right) \leq \lambda^{2}\left(1+2^{-4} R^{\beta}\right) d \tilde{\mu}_{i}\left(\tilde{\sigma}_{i}^{\prime}\right), i=1,2 .
$$


There is a small variation in the thickness of the third surface. A direct computation based on (3.9) gives

$$
T^{-1} \Sigma_{3}(\varepsilon) \subset \tilde{\Sigma}_{3}\left(\lambda^{-1}\left(1+2^{-8} R^{\beta}\right) \varepsilon\right) .
$$

Moreover, if $\tilde{\psi}(\cdot)=\psi(T \cdot)$, then

$$
\frac{\left(1-2^{-6} R^{\beta}\right)^{\frac{1}{2}}}{\lambda^{\frac{3}{2}}}\|\psi\|_{L^{2}\left(\mathbb{R}^{3}\right)} \leq\|\tilde{\psi}\|_{L^{2}\left(\mathbb{R}^{3}\right)} \leq \frac{\left(1+2^{-6} R^{\beta}\right)^{\frac{1}{2}}}{\lambda^{\frac{3}{2}}}\|\psi\|_{L^{2}\left(\mathbb{R}^{3}\right)} .
$$

From all the above considerations it follows that

$$
\begin{aligned}
& C(R, r, \varepsilon) \leq \\
& \quad \leq\left(1+2^{-4} R^{\beta}\right)^{\frac{5}{2}}\left(1-2^{-6} R^{\beta}\right)^{-\frac{1}{2}} C\left(r\left(1+R^{\beta}\right)^{1+\frac{1}{\beta}}, \varepsilon\left(1+2^{-8} R^{\beta}\right) \lambda^{-1}\right),
\end{aligned}
$$

and the bound (3.8) follows immediately since $R \leq 1$.

The following result establishes the key estimate needed for the induction on scales argument.

Proposition 3.4. Assume that $\left(2^{40} \varepsilon\right)^{\frac{2}{\beta+2}} \leq R$ and $R_{\beta}:=R^{\frac{\beta+2}{2(\beta+1)}}$. Then, the estimate

$$
C\left(R_{\beta}, \varepsilon\right) \leq\left(1+R^{\frac{\beta}{4}}\right) C\left(R_{\beta}, R, \varepsilon\right)
$$

holds true.

Proof. We split the proof in five steps.

Step 1 (Symmetrization). The numbers $C\left(R_{\beta}, \varepsilon\right)$ and $C\left(R, R_{\beta}, \varepsilon\right)$ are defined by (3.6) (with the additional constraints on $\Sigma_{i}$ ). That formula has the disadvantage of not revealing the symmetry of the problem with respect to the role of the three surfaces. However, (3.6) is equivalent to

$$
\left\langle f_{1} * f_{2}, f_{3}\right\rangle \leq C \sqrt{\varepsilon}\left\|f_{1}\right\|_{L^{2}\left(\Sigma_{1}\right)}\left\|f_{2}\right\|_{L^{2}\left(\Sigma_{2}\right)}\left\|f_{3}\right\|_{L^{2}\left(\Sigma_{3}(\varepsilon)\right)}
$$

for all $f_{3} \in L^{2}\left(\mathbb{R}^{3}\right)$. Upon replacing $f_{3}$ by $f_{3}(-\cdot)$ and $\Sigma_{3}$ by $-\Sigma_{3}$ and considering smooth, compactly supported $f_{3}$, this coincides with the triple convolution of the distributions $f_{1}, f_{2}$ with $f_{3}$ at zero, i.e.

$$
\left(f_{1} * f_{2} * f_{3}\right)(0) \leq C(R, \varepsilon) \sqrt{\varepsilon}\left\|f_{1}\right\|_{L^{2}\left(\Sigma_{1}\right)}\left\|f_{2}\right\|_{L^{2}\left(\Sigma_{2}\right)}\left\|f_{3}\right\|_{L^{2}\left(\Sigma_{3}(\varepsilon)\right)} .
$$

By density this is an equivalent definition of $C(R, \varepsilon)$. Since (3.16) is symmetric $^{2}$ in $\Sigma_{i}$ we prefer to use this as a definition of $C(R, \varepsilon)$. In a similar way we symmetrize the definition of $C\left(R_{\beta}, R, \varepsilon\right)$.

\footnotetext{
${ }^{2}$ up to the thickening of $\Sigma_{3}$, which is irrelevant at this scale.
} 
Step 2 (Reduction of scales). From now on we assume that $\Sigma_{i}$ are defined as in (3.3) where $\Omega_{i}$ are cubes of size $R_{\beta}$. By translation in the coordinate directions we may assume that $\phi_{i}\left(a_{i}^{0}\right)=0, i=1,2,3$. From (3.5) it follows for $r_{\beta}:=2^{-39} R^{\frac{\beta+2}{2}}$ that

$$
\Sigma_{i} \subset\left\{\left(x_{1}, x_{2}, x_{3}\right)|| x_{i} \mid \leq \frac{r_{\beta}}{2}\right\} \subset\left\{\left(x_{1}, x_{2}, x_{3}\right)|| x_{i} \mid \leq \frac{R}{8}\right\} .
$$

From $\left(2^{40} \varepsilon\right)^{\frac{2}{\beta+2}} \leq R$ it follows that $\varepsilon \leq \frac{r_{\beta}}{2}$, therefore

$$
\Sigma_{3}(\varepsilon) \subset\left\{\left(x_{1}, x_{2}, x_{3}\right)|| x_{3} \mid \leq r_{\beta}\right\} \subset\left\{\left(x_{1}, x_{2}, x_{3}\right)|| x_{3} \mid \leq \frac{R}{4}\right\} .
$$

Hence we are dealing with three scales ordered as follows:

$$
r_{\beta} \leq R \leq R_{\beta} .
$$

Step 3 (Decomposition of the surfaces). Inspired by (3.18) we make the following construction. We will recursively define an increasing sequence ${ }^{3}$ $\left(s_{k}\right)_{k \geq 0}$ with the properties

$$
s_{k+1} \in\left[s_{k}+\frac{1}{2} R, s_{k}+R\right]
$$

and

$$
\begin{aligned}
& \left\|f_{1}\right\|_{L^{2}\left(\Sigma_{1} \cap\left\{\left|x_{3}+s_{k+1}\right| \leq r_{\beta}\right\}\right)} \leq 2^{-17} R^{\frac{\beta}{4}}\left\|f_{1}\right\|_{L^{2}\left(\Sigma_{1} \cap\left\{\frac{1}{2} R \leq-x_{3}-s_{k} \leq R\right\}\right)}, \\
& \left\|f_{2}\right\|_{L^{2}\left(\Sigma_{2} \cap\left\{\left|x_{3}-s_{k+1}\right| \leq r_{\beta}\right\}\right)} \leq 2^{-17} R^{\frac{\beta}{4}}\left\|f_{2}\right\|_{L^{2}\left(\Sigma_{2} \cap\left\{\frac{1}{2} R \leq x_{3}-s_{k} \leq R\right\}\right)} .
\end{aligned}
$$

In order to do so, we set $s_{1}=-a-r_{\beta}$ for a number $a>0$ such that $f_{1}$ and $f_{2}$ have support in the slab $\mathbb{R}^{2} \times[-a, a]$. Then (3.20) is trivially verified for $k=0$. Assume we already have constructed $s_{k}$ for some $k \geq 0$. For $i=1,2$ the set $\left\{\frac{1}{2} R \leq(-1)^{i} x_{3}-s_{k} \leq R\right\} \subset \mathbb{R}^{3}$ comprises $m$ slabs $(-1)^{i} I_{1}, \ldots,(-1)^{i} I_{m}$ of thickness (in $x_{3}$-direction) $2 r_{\beta}$, where $m$ denotes the largest integer which is smaller or equal to the ratio $\frac{\frac{1}{2} R}{2 r_{\beta}}$. For the function

$$
\alpha_{i}=\left\|f_{i}\right\|_{L^{2}\left(\Sigma_{i} \cap\left\{\frac{1}{2} R \leq(-1)^{i} x_{3}-s_{k} \leq R\right\}\right)}^{-2} f_{i}^{2}
$$

it follows that

$$
\begin{aligned}
2 & \geq \sum_{l=1}^{m}\left(\int_{-I_{l} \cap \Sigma_{1}} \alpha_{1} d \mu_{1}+\int_{I_{l} \cap \Sigma_{2}} \alpha_{2} d \mu_{2}\right) \\
& \geq m \min _{l=1, \ldots, m}\left(\int_{-I_{l} \cap \Sigma_{1}} \alpha_{1} d \mu_{1}+\int_{I_{l} \cap \Sigma_{2}} \alpha_{2} d \mu_{2}\right) .
\end{aligned}
$$

\footnotetext{
${ }^{3}$ Notice that the sequence itself may depend on the functions $f_{1}$ and $f_{2}$, but the final bound will not depend on this sequence.
} 
This estimate implies that there exists $I_{l^{*}}$ such that

$$
\int_{(-1)^{i} I_{l^{*} \cap \Sigma_{i}}} f_{i}^{2} d \mu_{1} \leq \frac{2}{m}\left\|f_{i}\right\|_{L^{2}\left(\Sigma_{i} \cap\left\{\frac{1}{2} R \leq(-1)^{i} x_{3}-s_{k} \leq R\right\}\right)}^{2}, i=1,2 .
$$

We choose $s_{k+1}$ to be the center of $I_{l^{*}}$, which satisfies (3.19) because $I_{l^{*}} \subset$ $\left[s_{k}+\frac{1}{2} R, s_{k}+R\right]$ and (3.20) because $m^{-1} \leq 2^{-36} R^{\frac{\beta}{2}}$.

For this sequence we define

$$
\begin{aligned}
& \Sigma_{i}\left[k, \mathfrak{e}_{3}\right]=\Sigma_{i} \cap\left\{\left(x_{1}, x_{2}, x_{3}\right) \mid(-1)^{i} x_{3} \in\left[s_{k}+r_{\beta}, s_{k+1}-r_{\beta}\right]\right\}, \\
& \tilde{\Sigma}_{i}\left[k, \mathfrak{e}_{3}\right]=\Sigma_{i} \cap\left\{\left(x_{1}, x_{2}, x_{3}\right) \mid(-1)^{i} x_{3} \in\left[s_{k}-r_{\beta}, s_{k}+r_{\beta}\right]\right\} .
\end{aligned}
$$

With this notation it follows that

$$
\begin{aligned}
& s_{k}+\frac{1}{2} R \leq s_{k+1} \leq s_{k}+R \\
& \left\|f_{i}\right\|_{L^{2}\left(\tilde{\Sigma}_{i}\left[k, \mathfrak{e}_{3}\right]\right)} \leq 2^{-17} R^{\frac{\beta}{4}}\left\|f_{i}\right\|_{\left.L^{2}\left(\Sigma_{i}\left[k-1, \mathfrak{e}_{3}\right] \cup \tilde{\Sigma}_{i}\left[k, \mathfrak{e}_{3}\right]\right) \cup \Sigma_{i}\left[k, \mathfrak{e}_{3}\right]\right)}, \quad i=1,2 .
\end{aligned}
$$

Since (3.18) has the (more restrictive) analog (3.17) in all directions, we can perform a similar construction to define $\Sigma_{i}\left[k, \mathfrak{e}_{1}\right]$ and $\tilde{\Sigma}_{i}\left[k, \mathfrak{e}_{1}\right]$ for $i=2,3$ as well as $\Sigma_{i}\left[k, \mathfrak{e}_{2}\right]$ and $\tilde{\Sigma}_{i}\left[k, \mathfrak{e}_{2}\right]$ for $i=1,3$ with the same properties $(3.21)$ and (3.22). Moreover, we introduce

$$
\begin{aligned}
\Sigma_{1}^{k_{2}, k_{3}}= & \Sigma_{1}\left[k_{2}, \mathfrak{e}_{2}\right] \cap \Sigma_{1}\left[k_{3}, \mathfrak{e}_{3}\right], \\
\tilde{\Sigma}_{1}^{k_{2}, k_{3}}= & \left(\tilde{\Sigma}_{1}\left[k_{2}, \mathfrak{e}_{2}\right] \cap \Sigma_{1}\left[k_{3}, \mathfrak{e}_{3}\right]\right) \cup\left(\Sigma_{1}\left[k_{2}, \mathfrak{e}_{2}\right] \cap \tilde{\Sigma}_{1}\left[k_{3}, \mathfrak{e}_{3}\right]\right) \\
& \cup\left(\tilde{\Sigma}_{1}\left[k_{2}, \mathfrak{e}_{2}\right] \cap \tilde{\Sigma}_{1}\left[k_{3}, \mathfrak{e}_{3}\right]\right),
\end{aligned}
$$

and similarly $\Sigma_{2}^{k_{1}, k_{3}}, \tilde{\Sigma}_{2}^{k_{1}, k_{3}}$ and $\Sigma_{3}^{k_{1}, k_{2}}(\varepsilon), \tilde{\Sigma}_{3}^{k_{1}, k_{2}}(\varepsilon)$. Now, we have the decompositions

$$
\Sigma_{i}=\bigcup_{k, l} \Sigma_{i}^{k, l} \cup \tilde{\Sigma}_{i}^{k, l}, i=1,2
$$

and the same for $\Sigma_{3}(\varepsilon)$.

Step 4 (Properties of the new sets). In this step we collect three useful facts about our new sets.

a) Diameter: From (3.21) it follows that $\Sigma_{1}^{k_{2}, k_{3}}$, is generated as in (3.3) by $\Omega_{1}^{k_{2}, k_{3}} \subset C$, where $C$ is a cube of size $R$. In addition, since $\Omega_{1}^{k_{2}, k_{3}} \subset \Omega_{1}$, it follows that at the center $c_{0}$ of $C$ we have an estimate of type (3.7), namely

$$
\left|\nabla \phi_{1}\left(c_{0}\right)\right| \leq 2^{-40}\left(\sqrt{3} R_{\beta}\right)^{\beta} .
$$

A similar characterization holds true for $\tilde{\Sigma}_{1}^{k_{2}, k_{3}}, \Sigma_{2}^{k_{1}, k_{3}}$, etc. This basically says that if in (3.16) we replace each $\Sigma_{i}$ by $\Sigma_{i}^{k_{l}, k_{m}}$ or $\tilde{\Sigma}_{i}^{k_{l}, k_{m}}$, then the constant should be adjusted to $C\left(R_{\beta}, R, \varepsilon\right)$. 
b) Orthogonality: The reason to introduce the decompositions from the previous step is to apply almost orthogonality arguments. More exactly we claim that

$$
\left(\left.\left.\left.f_{1}\right|_{\Sigma_{1}^{k_{2}, k_{3}}} * f_{2}\right|_{\Sigma_{2}^{k_{1}, k_{3}^{\prime}}} * f_{3}\right|_{\Sigma_{3}^{k_{1}^{\prime}, k_{2}^{\prime}}(\varepsilon)}\right)(0)=0
$$

unless $k_{i}=k_{i}^{\prime}$ for $i=1,2,3$.

Indeed, by definition of $\Sigma_{1}^{k_{2}, k_{3}}$ and $\Sigma_{2}^{k_{1}, k_{3}^{\prime}}$ we have

$$
\begin{aligned}
\operatorname{supp}\left(\left.\left.f_{1}\right|_{\Sigma_{1}^{k_{2}, k_{3}}} * f_{2}\right|_{\Sigma_{2}^{k_{1}, k_{3}^{\prime}}}\right) \subset & \\
& \subset\left\{\left(x_{1}, x_{2}, x_{3}\right) \mid x_{3} \in\left[s_{k_{3}^{\prime}}-s_{k_{3}+1}+2 r_{\beta}, s_{k_{3}^{\prime}+1}-s_{k_{3}}-2 r_{\beta}\right]\right\} .
\end{aligned}
$$

For the left hand side of (3.23) to be different from zero it is necessary that

$$
\left[s_{k_{3}^{\prime}}-s_{k_{3}+1}+2 r_{\beta}, s_{k_{3}^{\prime}+1}-s_{k_{3}}-2 r_{\beta}\right] \cap\left[-r_{\beta}, r_{\beta}\right] \neq 0 .
$$

due to (3.18), which leads to $k_{3}=k_{3}^{\prime}$ because $\left(s_{k}\right)$ is strictly increasing. In a similar manner it follows that $k_{i}=k_{i}^{\prime}$ for $i=1,2$.

A similar argument, using the properties of $\left(s_{k}\right)_{k \geq 1}$, provides that if one allows in (3.23) one or more of the $\Sigma_{i}$ to be replaced by the corresponding set $\tilde{\Sigma}_{i}$, the convolution is zero unless $\left|k_{i}-k_{i}^{\prime}\right| \leq 1$.

c) Smallness on $\tilde{\Sigma}$ : The lack of perfect orthogonality in (3.23) when $\tilde{\Sigma}$ 's are involved is compensated by the following smallness of mass on those sets

$$
\left(\sum_{k, l}\left\|f_{i}\right\|_{L^{2}\left(\tilde{\Sigma}_{i}^{k, l}\right)}^{2}\right)^{\frac{1}{2}} \leq 2^{-16} R^{\frac{\beta}{4}}\left\|f_{i}\right\|_{L^{2}\left(\Sigma_{i}\right)}
$$

We prove (3.24) for $i=1$ since the other cases are similar. From the definitions of the sets we have the straightforward estimate

$$
\sum_{k_{2}, k_{3}}\left\|f_{1}\right\|_{L^{2}\left(\tilde{\Sigma}_{1}^{k_{2}, k_{3}}\right)}^{2} \leq \sum_{k_{2}}\left\|f_{1}\right\|_{L^{2}\left(\tilde{\Sigma}_{1}\left[k_{2}, \mathfrak{e}_{2}\right]\right)}^{2}+\sum_{k_{3}}\left\|f_{1}\right\|_{L^{2}\left(\tilde{\Sigma}_{1}\left[k_{3}, \mathfrak{e}_{3}\right]\right)}^{2}
$$

Then one uses (3.22) and the analog of (3.22) for $\tilde{\Sigma}_{1}\left[k_{2}, \mathfrak{e}_{2}\right]$ to estimate each term by $2^{-34} R^{\frac{\beta}{2}}\left\|f_{1}\right\|_{L^{2}\left(\Sigma_{1}\right)}^{2}$ and obtains (3.24).

Step 5 (Conclusion of the proof). Based on the above analysis on sets we decompose

$$
\left(\left.\left.\left.f_{1}\right|_{\Sigma_{1}} * f_{2}\right|_{\Sigma_{2}} * f_{3}\right|_{\Sigma_{3}(\varepsilon)}\right)(0)=S+T
$$

where

$$
S=\left.\left.\left.\sum_{k_{1}, k_{2}, k_{3}, k_{1}^{\prime}, k_{2}^{\prime}, k_{3}^{\prime}} f_{1}\right|_{\Sigma_{1}^{k_{2}, k_{3}}} * f_{2}\right|_{\Sigma_{2}^{k_{1}, k_{3}^{\prime}}} * f_{3}\right|_{\Sigma_{3}^{k_{1}^{\prime}, k_{2}^{\prime}}(\varepsilon)}(0)
$$

where the remainder $T$ contains 7 sums of the same type as $S$, except that one $(3$ cases $)$, two $(3$ cases $)$ or all three $(1$ case) $\Sigma$ are replaced by $\tilde{\Sigma}$. 
We decompose as

$$
T=T_{1}+T_{2}+T_{3}+T_{12}+T_{13}+T_{23}+T_{123}
$$

where the subscripts indicate the positions of the $\tilde{\Sigma}$ factors.

On behalf of the orthogonality relation (3.23) we observe that

$$
S=\left.\left.\left.\sum_{k_{1}, k_{2}, k_{3}} f_{1}\right|_{\Sigma_{1}^{k_{2}, k_{3}}} * f_{2}\right|_{\Sigma_{2}^{k_{1}, k_{3}}} * f_{3}\right|_{\Sigma_{3}^{k_{1}, k_{2}}(\varepsilon)}(0) .
$$

From the conclusions in Step 4 a) above, we obtain

$$
\begin{aligned}
S & \leq C\left(R_{\beta}, R, \varepsilon\right) \sum_{k_{1}, k_{2}, k_{3}}\left\|f_{1}\right\|_{L^{2}\left(\Sigma_{1}^{k_{2}, k_{3}}\right)}\left\|f_{2}\right\|_{L^{2}\left(\Sigma_{2}^{k_{1}, k_{3}}\right)}\left\|f_{3}\right\|_{L^{2}\left(\Sigma_{3}^{k_{1}, k_{2}}(\varepsilon)\right)} \\
& \leq C\left(R_{\beta}, R, \varepsilon\right)\left\|f_{1}\right\|_{L^{2}\left(\Sigma_{1}\right)}\left\|f_{2}\right\|_{L^{2}\left(\Sigma_{2}\right)}\left\|f_{3}\right\|_{L^{2}\left(\Sigma_{3}(\varepsilon)\right)}
\end{aligned}
$$

In passing to the last line we have used the Cauchy-Schwartz inequality with respect to all three $k_{i}$ 's and the fact that the sets $\Sigma_{1}^{k_{2}, k_{3}}$ are disjoint with respect to the pair $\left(k_{2}, k_{3}\right)$ (and the same for the sets $\Sigma_{2}^{k_{1}, k_{3}}, \Sigma_{3}^{k_{1}, k_{2}}$ ).

For each term in the remainder the same argument applies up to the orthogonality issue. By the almost orthogonality the first term in the remainder is given by

$$
T_{1}=\sum_{*}\left(\left.\left.\left.f_{1}\right|_{\tilde{\Sigma}_{1}^{k_{2}, k_{3}}} * f_{2}\right|_{\Sigma_{2}^{k_{1}, k_{3}^{\prime}}} * f_{3}\right|_{\Sigma_{3}^{k_{1}^{\prime}, k_{2}^{\prime}}(\varepsilon)}\right)(0)
$$

where $*$ indicates summation with respect to $k_{1}, k_{2}, k_{3}, k_{1}^{\prime}, k_{2}^{\prime}, k_{3}^{\prime}$ satisfying $\left|k_{i}-k_{i}^{\prime}\right| \leq 1$ for $i=1,2,3$. We obtain

$$
\begin{aligned}
T_{1} & \leq C\left(R_{\beta}, R, \varepsilon\right) \sum_{*}\left\|f_{1}\right\|_{L^{2}\left(\tilde{\Sigma}_{1}^{k_{2}, k_{3}}\right)}\left\|f_{2}\right\|_{L^{2}\left(\Sigma_{2}^{\left.k_{1}, k_{3}^{\prime}\right)}\right.}\left\|f_{3}\right\|_{L^{2}\left(\Sigma_{3}^{k_{1}^{\prime}, k_{2}^{\prime}}(\varepsilon)\right)} \\
& \leq 27 C\left(R_{\beta}, R, \varepsilon\right)\left(\sum_{k_{2}, k_{3}}\left\|f_{1}\right\|_{L^{2}\left(\tilde{\Sigma}_{1}^{k_{2}, k_{3}}\right)}^{2}\right)^{\frac{1}{2}}\left\|f_{2}\right\|_{L^{2}\left(\Sigma_{2}\right)}\left\|f_{3}\right\|_{L^{2}\left(\Sigma_{3}(\varepsilon)\right)} \\
& \leq 2^{-11} R^{\frac{\beta}{4}} C\left(R_{\beta}, R, \varepsilon\right)\left\|f_{1}\right\|_{L^{2}\left(\Sigma_{1}\right)}\left\|f_{2}\right\|_{L^{2}\left(\Sigma_{2}\right)}\left\|f_{3}\right\|_{L^{2}\left(\Sigma_{3}(\varepsilon)\right)} .
\end{aligned}
$$

where we have used (3.24) in passing to the last line. If one considers the remaining terms in (3.26) the same estimate holds true, which amounts to

$$
T \leq R^{\frac{\beta}{4}} C\left(R_{\beta}, R, \varepsilon\right)\left\|f_{1}\right\|_{L^{2}\left(\Sigma_{1}\right)}\left\|f_{2}\right\|_{L^{2}\left(\Sigma_{2}\right)}\left\|f_{3}\right\|_{L^{2}\left(\Sigma_{3}(\varepsilon)\right)}
$$

This estimate for the remainder term $T$ together with the estimate for the main term $S$ and (3.25) leads to (3.15). 
As a corollary we obtain

Corollary 3.5. Under the assumption $\left(2^{40} \varepsilon\right)^{\frac{2}{\beta+2}} \leq R$ the estimate

$$
C\left(R_{\beta}, \varepsilon\right) \leq\left(1+R_{\beta}^{\frac{\beta}{4}}\right)^{2} C\left(\left(1+R_{\beta}^{\beta}\right)^{1+\frac{1}{\beta}} R, \varepsilon\left(1+R_{\beta}^{\beta}\right)^{1+\frac{1}{\beta}}\right)
$$

holds true, where $R_{\beta}$ is defined as $R_{\beta}=R^{\frac{\beta+2}{(\beta+1)}}$.

Proof. This result is a direct consequence of (3.15) and (3.8).

We can now proceed with the proof of the result claimed in (3.2).

Proof of Proposition 3.1. We recursiveley define $R(k)$ as

$$
R(k+1)=R(k)^{\frac{2(\beta+1)}{\beta+2}}\left(1+R(k)^{\beta}\right)^{1+\frac{1}{\beta}} .
$$

A straightforward computation gives

$$
\frac{R(k+1)}{R(k)}=R(k)^{\frac{\beta}{\beta+2}}\left(1+R(k)^{\beta}\right)^{1+\frac{1}{\beta}} .
$$

Since the right-hand side is an increasing function in $R(k)$, one can choose $R(0)=C_{\beta}$ such that additionally

$$
R(0)^{\frac{\beta}{\beta+2}}\left(1+R(0)^{\beta}\right)^{1+\frac{1}{\beta}} \leq \frac{1}{2}
$$

is satisfied. With this choice the sequence $R(k)$ is strictly decreasing and $\lim _{k \rightarrow \infty} R(k) \leq \lim _{k \rightarrow \infty} 2^{-k}=0$. The result in (3.28) provides

$$
C(R(k), \varepsilon) \leq\left(1+R(k)^{\frac{\beta}{4}}\right)^{2} C\left(R(k+1), \varepsilon\left(1+R(k)^{\beta}\right)^{1+\frac{1}{\beta}}\right) .
$$

However, in order to apply the above inequality we need to verify the required bounds. If

$$
\varepsilon(k)=\varepsilon \prod_{l=0}^{k-1}\left(1+R(l)^{\beta}\right)^{1+\frac{1}{\beta}},
$$

then the above formula can be used as long as $\left(2^{40} \varepsilon(k)\right)^{\frac{2}{\beta+2}} \leq R(k)$. For $k=0$ one needs to verify $\left(2^{40} \varepsilon\right)^{\frac{2}{\beta+2}} \leq C_{\beta}$ for which it is enough to take $\varepsilon \leq \varepsilon_{\beta}=2^{-40} C_{\beta}^{\frac{\beta+2}{2}}$. For $k \geq 1$ we have that

$$
\varepsilon(k)^{\frac{2}{\beta+2}}=\varepsilon^{\frac{2}{\beta+2}}\left(\prod_{l=0}^{k-1}\left(1+R(l)^{\beta}\right)\right)^{\frac{2}{\beta+2}\left(1+\frac{1}{\beta}\right)}
$$

is an increasing sequence, therefore we can find $N$ to be the highest value of $k$ with the property $\varepsilon(k)^{\frac{2}{\beta+2}} \leq R(k)$. 
Now we can use (3.30) for all $k \leq N$ and by iterating it, we obtain

$$
C(R(0), \varepsilon) \leq \prod_{k=0}^{N-1}\left(1+R(k)^{\frac{\beta}{4}}\right)^{2} C(R(N), \varepsilon(N)) .
$$

Using (3.29) we estimate

$$
\ln \prod_{k=0}^{\infty}\left(1+R(k)^{\beta}\right)^{1+\frac{1}{\beta}} \leq\left(1+\frac{1}{\beta}\right) \sum_{k=0}^{\infty} R(k)^{\beta} \leq\left(1+\frac{1}{\beta}\right) R(0)^{\beta} \sum_{k=0}^{\infty}\left(\frac{1}{2^{\beta}}\right)^{k},
$$

which is less than $\ln (2)$ by making $C_{\beta}$ small enough, which shows that $\prod_{k=0}^{\infty}\left(1+R(k)^{\beta}\right)^{1+\frac{1}{\beta}} \leq 2$ and therefore $\varepsilon(k) \leq 2 \varepsilon$ for all $k \geq 0$.

Since $R(N+1) \leq\left(2^{40} \varepsilon(N+1)\right)^{\frac{2}{\beta+2}} \leq\left(2^{41} \varepsilon(N)\right)^{\frac{2}{\beta+2}}$, it follows that $R(N) \leq 2^{41}(\varepsilon(N))^{\frac{1}{\beta+1}} \leq\left(2^{41} \varepsilon(N)\right)^{\frac{1}{\beta+1}}$. Now we can apply the result in Lemma 3.2 and obtain

$$
C(R(N), \varepsilon(N)) \leq C\left(R(N), 2^{41} \varepsilon(N)\right) \leq \sqrt{2^{41} \varepsilon(N)} \leq 2^{21} \sqrt{\varepsilon} .
$$

In a similar manner as above we obtain

$$
\ln \prod_{k=0}^{\infty}\left(1+R(k)^{\frac{\beta}{4}}\right)^{2} \leq 1
$$

at the expense of adjusting $C_{\beta}$ again. The last estimate together with (3.31) and (3.32) proves that

$$
C(R(0), \varepsilon) \leq C \sqrt{\varepsilon}
$$

for all $\varepsilon \leq \varepsilon_{\beta}$. From this the claim in Proposition 3.1 follows by partitioning each $\Sigma_{i}$ into a finite number of pieces of diameter less than $R(0)$ and, in addition, by partitioning $\Sigma_{3}(\varepsilon)$ into a finite number of pieces $\Sigma_{3}^{\prime}\left(\varepsilon_{\beta}\right)$ where $\Sigma_{3}^{\prime}$ are translates of $\Sigma_{3}$ in the $z$-direction.

Acknowledgements. The authors are grateful to Terence Tao for pointing out the references $[4,5]$, and to Anthony Carbery for his helpful comments on an earlier version of this paper.

\section{References}

[1] Bejenaru, I.: Quadratic nonlinear derivative Schrödinger equations. II. Trans. Amer. Math. Soc. 360 (2008), no. 11, 5925-5957.

[2] Bejenaru, I. And De Silva, D.: Low regularity solutions for a 2D quadratic nonlinear Schrödinger equation. Trans. Amer. Math. Soc. 360 (2008), no. 11, 5805-5830.

[3] Bejenaru, I., Herr, S., Holmer, J. and Tataru, D.: On the 2D Zakharov system with $L^{2}$-Schrödinger data. Nonlinearity 22 (2009), no. 5, 1063-1089. 
[4] Bennett, J., Carbery, A. and Tao, T.: On the multilinear restriction and Kakeya conjectures. Acta Math. 196 (2006), no. 2, 261-302.

[5] Bennett, J., Carbery, A. and Wright, J.: A non-linear generalisation of the Loomis-Whitney inequality and applications. Math. Res. Lett. 12 (2005), no. 4, 443-457.

[6] Colliander, J. E., Delort, J.-M., Kenig, C. E. and Staffilani, G.: Bilinear estimates and applications to 2D NLS. Trans. Amer. Math. Soc. 353 (2001), no. 8, 3307-3325 (electronic).

[7] Ionescu, A. D., Kenig, C. E. And Tataru, D.: Global well-posedness of the KP-I initial-value problem in the energy space. Invent. Math. 173 (2008), no. 2, 265-304.

[8] Loomis, L. H. And Whitney, H.: An inequality related to the isoperimetric inequality. Bull. Amer. Math. Soc. 55 (1949), 961-962.

[9] TAO, T.: Multilinear weighted convolution of $L^{2}$-functions, and applications to nonlinear dispersive equations. Amer. J. Math. 123 (2001), no. 5, 839-908.

Recibido: 2 de octubre de 2008

$$
\begin{array}{r}
\text { Ioan Bejenaru } \\
\text { Department of Mathematics } \\
\text { University of Chicago } \\
\text { Chicago, IL 60637, USA } \\
\text { bejenaru@math.uchicago.edu } \\
\text { Sebastian Herr } \\
\text { Department of Mathematics } \\
\text { University of California } \\
\text { Berkeley, CA 94720-3840, USA } \\
\text { Current address: } \\
\text { Mathematisches Institut } \\
\text { Universität Bonn } \\
\text { Endenicher Allee 60, 53115 Bonn, Germany } \\
\text { herr@math. uni-bonn.de } \\
\text { Daniel Tataru } \\
\text { Department of Mathematics } \\
\text { University of California }
\end{array}
$$

I. B. was supported in part by NSF grant DMS0738442, S. H. acknowledges support from NSF grant DMS0354539 and D. T. was supported in part by NSF grant DMS0801261. 